\title{
A characterization of positive normal functionals on the full operator algebra
}

\author{
Zoltán Sebestyén, Zsigmond Tarcsay and Tamás Titkos
}

\begin{abstract}
Using the recent theory of Krein-von Neumann extensions for positive functionals we present several simple criteria to decide whether a given positive functional on the full operator algebra $B(H)$ is normal. We also characterize those functionals defined on the left ideal of finite rank operators that have a normal extension.
\end{abstract}

Mathematics Subject Classification (2010). Primary 46K10, 46A22.

Keywords. Krein-von Neumann extension, Normal functionals, Trace.

The aim of this short note is to present a theoretical application of the generalized Krein-von Neumann extension, namely to offer a characterization of positive normal functionals on the full operator algebra. To begin with, let us fix our notations. Given a complex Hilbert space $H$, denote by $B(H)$ the full operator algebra, i.e., the $C^{*}$-algebra of continuous linear operators on $H$. The symbols $B_{F}(H), B_{1}(H), B_{2}(H)$ are referring to the ideals of continuous finite rank operators, trace class operators, and Hilbert-Schmidt operators, respectively. Recall that $B_{2}(H)$ is a complete Hilbert algebra with respect to the inner product

$$
(X \mid Y)_{2}=\operatorname{Tr}\left(Y^{*} X\right)=\sum_{e \in \mathcal{E}}(X e \mid Y e), \quad X, Y \in B_{2}(H) .
$$

Here Tr refers to the the trace functional and $\mathcal{E}$ is an arbitrary orthonormal basis in $H$. Recall also that $B_{1}(H)$ is a Banach ${ }^{*}$-algebra under the norm $\|X\|_{1}:=\operatorname{Tr}(|X|)$, and that $B_{F}(H)$ is dense in both $B_{1}(H)$ and $B_{2}(H)$, with respect to the norms $\|\cdot\|_{1}$ and $\|\cdot\|_{2}$, respectively. It is also known that $X \in B_{1}(H)$ holds if and only if $X$ is the product of two elements of $B_{2}(H)$. For the proofs and further basic properties of Hilbert-Schmidt and trace class operators we refer the reader to 1 .

The first author Zsigmond Tarcsay was supported by the Hungarian Ministry of Human Capacities, NTP-NFT-17. Corresponding author: Tamás Titkos. 
Let $\mathscr{A}$ be a von Neumann algebra, that is a strongly closed *-subalgebra of $B(H)$ containing the identity. A bounded linear functional $f: \mathscr{A} \rightarrow \mathbb{C}$ is called normal if it is continuous in the ultraweak topology, that is $f$ belongs to the predual of $\mathscr{A}$. It is well known that the predual of $B(H)$ is $B_{1}(H)$, hence every normal functional can be represented by a trace class operator. We will use this property as the definition.

Definition. A linear functional $f: B(H) \rightarrow \mathbb{C}$ is called a normal functional if there exists a trace class operator $F$ such that

$$
f(X):=\operatorname{Tr}(X F)=\operatorname{Tr}(F X), \quad X \in B(H) .
$$

Remark that such a functional is always continuous due to the inequality

$$
|\operatorname{Tr}(X F)| \leq\|F\|_{1} \cdot\|X\| \text {. }
$$

Our main tool is a canonical extension theorem for linear functionals which is analogous with the well-known operator extension theorem named after the pioneers of the 20th century operator theory M.G. Krein [2] and J. von Neumann [3]. For the details see Section 5 in [5], especially Theorem 5.6 and the subsequent comments. Let us recall the cited theorem:

A Krein-von Neumann type extension. Let $\mathscr{I}$ be a left ideal of the complex Banach ${ }^{*}$-algebra $\mathscr{A}$, and consider a linear functional $\varphi: \mathscr{I} \rightarrow \mathbb{C}$. The following statements are equivalent:

(a) There is a representable positive functional $\varphi^{\bullet}: \mathscr{A} \rightarrow \mathbb{C}$ extending $\varphi$, which is minimal in the sense that

$$
\varphi^{\bullet}\left(x^{*} x\right) \leq \widetilde{\varphi}\left(x^{*} x\right), \quad \text { holds for all } x \in \mathscr{A},
$$

whenever $\widetilde{\varphi}: \mathscr{A} \rightarrow \mathbb{C}$ is a representable extension of $\varphi$.

(b) There is a constant $C \geq 0$ such that $|\varphi(a)|^{2} \leq C \cdot \varphi\left(a^{*} a\right)$ for all $a \in \mathscr{I}$.

We remark that the construction used in the proof of the above theorem is closely related to the one developed in [4 for Hilbert space operators. The main advantage of that construction is that we can compute the values of the smallest extension $\varphi^{\bullet}$ on positive elements, namely

$$
\varphi^{\bullet}\left(x^{*} x\right)=\sup \left\{\left|\varphi\left(x^{*} a\right)\right|^{2} \mid a \in \mathscr{I}, \varphi\left(a^{*} a\right) \leq 1\right\} \quad \text { for all } x \in \mathscr{A} .
$$

The minimal extension $\varphi^{\bullet}$ is called the Krein-von Neumann extension of $\varphi$.

The characterization we are going to prove is stated as follows.

Main Theorem. For a given positive functional $f: B(H) \rightarrow \mathbb{C}$ the following statements are equivalent:

(i) $f$ is normal.

(ii) There exists a normal positive functional $g$ such that $f \leq g$.

(iii) $f \leq g$ holds for any positive functional $g$ that agrees with $f$ on $B_{F}(H)$.

(iv) For any $X \in B(H)$ we have

$$
f\left(X^{*} X\right)=\sup \left\{\left|f\left(X^{*} A\right)\right|^{2} \mid A \in B_{F}(H), f\left(A^{*} A\right) \leq 1\right\} .
$$

(v) $f(I) \leq \sup \left\{|f(A)|^{2} \mid A \in B_{F}(H), f\left(A^{*} A\right) \leq 1\right\}$. 
Proof. The proof is divided into three claims, which might be interesting on their own right. Before doing that we make some observations. For a given trace class operator $S$ let us denote by $f_{S}$ the normal functional defined by

$$
f_{S}(X):=\operatorname{Tr}(X S), \quad X \in B(H) .
$$

The map $S \mapsto f_{S}$ is order preserving between positive trace class operators and normal positive functionals. Indeed, if $S \geq 0$ then

$$
f_{S}\left(A^{*} A\right)=\operatorname{Tr}\left(A^{*} A S\right)=\left\|A S^{1 / 2}\right\|_{2}^{2} \geq 0 .
$$

Conversely, if $f_{S}$ is a positive functional and $P_{\langle h\rangle}$ denotes the orthogonal projection onto the subspace spanned by $h \in H$, we obtain $S \geq 0$ by

$$
(S h \mid h)=\operatorname{Tr}\left(P_{\langle h\rangle} S\right)=f_{S}\left(P_{\langle h\rangle}^{*} P_{\langle h\rangle}\right) \geq 0, \quad \text { for all } h \in H .
$$

Our first two claims will prove that (i) and (iv) are equivalent.

Claim 1. Let $f$ be a normal positive functional and set $\varphi:=\left.f\right|_{B_{F}(H)}$. Then $f$ is the smallest positive extension of $\varphi$, i.e $\varphi^{\bullet}=f$.

Proof of Claim 1. Since $f \geq 0$ is normal, there is a positive $S \in B_{1}(H)$ such that $f=f_{S}$. By assumption $\varphi$ has a positive extension (namely $f$ itself is one), thus there exists also the Krein-von Neumann extension denoted by $\varphi^{\bullet}$. As $f_{S}-\varphi^{\bullet}$ is a positive functional due to the minimality of $\varphi^{\bullet}$, its norm is attained at identity $I$. Therefore it is enough to show that

$$
\varphi^{\bullet}(I) \geq f_{S}(I)=\operatorname{Tr}(S) .
$$

We know from (荬) that

$$
\varphi^{\bullet}\left(X^{*} X\right)=\sup \left\{\left|\varphi\left(X^{*} A\right)\right|^{2} \mid A \in B_{F}(H), \varphi\left(A^{*} A\right) \leq 1\right\}
$$

for any $X \in B(H)$. Choosing $A=\operatorname{Tr}(S)^{-1 / 2} P$ for any projection $P$ with finite rank, we see that $\varphi\left(A^{*} A\right)=\operatorname{Tr}(S)^{-1} \operatorname{Tr}(P S) \leq 1$, whence

$$
\varphi^{\bullet}(I) \geq|\varphi(A)|^{2}=\frac{\operatorname{Tr}(P S)^{2}}{\operatorname{Tr}(S)} .
$$

Taking supremum in $P$ on the right hand side we obtain $\varphi^{\bullet}(I) \geq \operatorname{Tr}(S)$, which proves the claim.

Claim 2. The smallest positive extension of $\varphi$, i.e. $\left(\left.f\right|_{B_{F}(H)}\right)$ is normal.

Proof of Claim 2. First observe that the restriction of $f$ to $B_{2}(H)$ defines a continuous linear functional on $B_{2}(H)$ with respect to the norm $\|\cdot\|_{2}$. Due to the Riesz representation theorem, there exists a unique representing operator $S \in B_{2}(H)$ such that

$$
f(A)=(A \mid S)_{2}=\operatorname{Tr}\left(S^{*} A\right), \quad \text { for all } A \in B_{2}(H) .
$$

We are going to show that $S \in B_{1}(H)$. Indeed, let $\mathcal{E}$ be an orthonormal basis in $H$ and let $\mathcal{F}$ be any non-empty finite subset of $\mathcal{E}$. Denoting by $P_{\mathcal{F}}$ the orthogonal projection onto the subspace spanned by $\mathcal{F}$ we get

$$
\sum_{e \in \mathcal{F}}(S e \mid e)=\left(P_{\mathcal{F}} \mid S\right)_{2}=f\left(P_{\mathcal{F}}\right) \leq f(I) .
$$


Taking supremum in $\mathcal{F}$ we obtain that $S$ is in trace class. By Claim 1 , the smallest positive extension $\varphi^{\bullet}$ of $\varphi$ equals $f_{S}$ which is normal. This proves Claim 2.

Now, we are going to prove (ii) $\Rightarrow(\mathrm{i})$.

Claim 3. If there exists a normal positive functional $g$ such that $f \leq g$ holds, then $f$ is normal as well.

Proof of Claim 3. Let $g$ be a normal positive functional dominating $f$, and let $T$ be a trace class operator such that $g=f_{T}$. According to Claim 2 it is enough to prove that $f=\varphi^{\bullet}$. Since $h:=f-\varphi^{\bullet}$ is positive, this will follow by showing that $h(I)=0$. We see from (***) that $h(A)=0$ for any finite rank operator $A$. Consequently, as $h \leq f \leq f_{T}$, it follows that

$$
h(I)=h(I-P) \leq f_{T}(I-P)=\operatorname{Tr}(T)-\operatorname{Tr}(T P),
$$

for any finite rank projection $P$. Taking infimum in $P$ we obtain $h(I)=0$ and therefore Claim 3 is established.

Completing the proof we mention all the missing trivial implications. Taking $g:=f$, (i) implies (ii). As (氶) means that $\varphi^{\bullet}=f$, equivalence of (iii) and (iv) follows from the minimality of the Krein-von Neumann extension. Replacing $X$ with $I$ in (买) we obtain (v). Conversely, (v) implies (iv) as $\varphi^{\bullet} \leq f$ and $f-\varphi^{\bullet}$ attains its norm at $I$.

Finally, we remark that the above proof contains a characterization of having normal extension for a functional defined on $B_{F}(H)$.

Corollary. Let $\varphi: B_{F}(H) \rightarrow \mathbb{C}$ be a linear functional. The following statements are equivalent to the existence of a normal extension.

(a) There is a $C \geq 0$ such that $|\varphi(A)|^{2} \leq C \cdot \varphi\left(A^{*} A\right)$ for all $A \in B_{F}(H)$.

(b) There is a positive functional $f$ such that $\left.f\right|_{B_{F}(H)}=\varphi$.

(c) There is an $F \in B_{1}(H)$ such that $\varphi(A)=\operatorname{Tr}(F A)$ for all $A \in B_{F}(H)$.

\section{References}

[1] R. V. Kadison and J. R. Ringrose, Fundamentals of the theory of operator algebras I., Academic Press, New York, 1983.

[2] M. G. Krein, The theory of self-adjoint extensions of semi-bounded Hermitian transformations and its applications, I-II, Mat. Sbornik 20, 431-495, Mat. Sbornik 21, 365-404 (1947) (Russian)

[3] J. von Neumann, Allgemeine Eigenwerttheorie Hermitescher Funktionaloperatoren, Math. Ann., 102 (1930) 49-131.

[4] Z. Sebestyén, Operator extensions on Hilbert space, Acta Sci. Math. (Szeged), 57 (1993), 233-248.

[5] Z. Sebestyén, Zs. Szücs, and Zs. Tarcsay, Extensions of positive operators and functionals, Linear Algebra Appl., 472 (2015), 54-80. 


\author{
Zoltán Sebestyén \\ Department of Applied Analysis \\ Eötvös Loránd University \\ Pázmány Péter sétány $1 / \mathrm{c}$. \\ Budapest H-1117 \\ Hungary \\ e-mail: sebesty@cs.elte.hu

\section{Zsigmond Tarcsay} \\ Department of Applied Analysis \\ Eötvös Loránd University \\ Pázmány Péter sétány 1/c. \\ Budapest H-1117 \\ Hungary \\ e-mail: tarcsay@cs.elte.hu \\ Tamás Titkos \\ Alfréd Rényi Institute of Mathematics \\ Hungarian Academy of Sciences \\ Reáltanoda utca 13-15. \\ Budapest H-1053 \\ Hungary \\ e-mail: titkos.tamas@renyi.mta.hu
}

